This paper was retracted on November 4, 2021.

\title{
Magnetic Hysteresis of Single-Molecule Magnets Adsorbed on Ferromagnetic Substrate
}

Zhi Kun Qi, ${ }^{\dagger}$ Puneet Mishra, ${ }^{*},{ }^{\ddagger} \S^{\circ}$ Ferdous Ara, ${ }^{\dagger}$ Hirofumi Oka, $^{\ddagger}, \|$ Yasuyuki Sainoo, ${ }^{\ddagger}$ Keiichi Katoh, Masahiro Yamashita, ${ }^{*},, \|, \perp$ and Tadahiro Komeda ${ }^{*}, * 0$

\footnotetext{
${ }^{\dagger}$ Department of Chemistry, Graduate School of Science, Tohoku University, Aramaki-AzaAoba, Aoba-Ku, Sendai 980-8578, Japan

${ }^{ \pm}$Institute of Multidisciplinary Research for Advanced Materials, Tohoku University, Katahira 2-1-1, Aoba-ku, Sendai 980-8577, Japan

${ }^{\S}$ Department of Physics, Central University of South Bihar, Gaya-824236, India

"WPI Research Center, Advanced Institute for Materials Research, Tohoku University, 2-1-1 Katahira, Aoba-Ku, Sendai 980-8577, Japan

${ }^{\perp}$ School of Materials Science and Engineering, Nankai University, Tianjin 300350, China
}

ABSTRACT: Single-molecule magnets (SMMs), exhibiting magnetic bistability and a large energy barrier to the magnetization relaxation, are attractive candidates for realizing efficient spintronic devices such as ultra-highdensity memories and quantum computers. Here, we demonstrate robust spin-polarization of a quintessential SMM, the double-decker bis(phthalocyaninato)terbium(III) $\left(\mathrm{TbPc}_{2}\right)$ molecules, adsorbed on the bilayer $\mathrm{Co}$ islands on $\mathrm{Au}(111)$ using spin-polarized scanning tunneling microscopy, visualizing the switching of the SMM spin

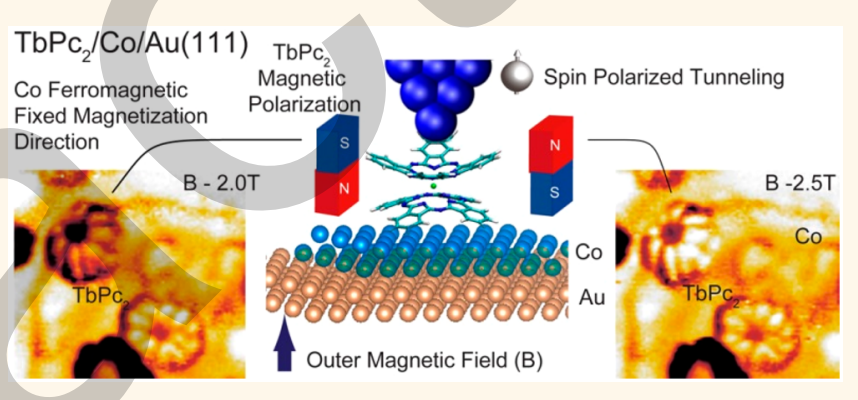
with submolecular resolution. Our results reveal that molecular spins of $\mathrm{TbPc}_{2}$ are antiferromagnetically coupled to the magnetization of the Co island, irrespective of the bonding configuration of the molecule. Stable spin-polarization is inferred from the substantial hysteresis observed in the magnetization curves measured over the molecules. This is in contrast to the reports of near-zero remanence for the bulk $\mathrm{TbPc}_{2}$ crystals and the $\mathrm{TbPc}_{2}$ molecules on a nonmagnetic substrate. The observation of a significant hysteretic opening can be attributed to the large magnetic anisotropy barrier of the molecule and the suppression of the quantum tunneling process. We anticipate that our approach of using the substrate to control and enhance the properties of a $\mathrm{TbPc}_{2} \mathrm{SMM}$ will accelerate further research toward realization of SMM-based functional spintronic devices.

KEYWORDS: single-molecule magnet, spin-polarized scanning tunneling microscopy, quantum tunneling, antiferromagnetic coupling, magnetic hysteresis

r he persistent trend of miniaturization has led to the exploration of magnetic nanostructures with everdecreasing feature size and large magnetic anisotropy for developing ultra-high-density storage devices and for implementing quantum computation. In this context, individual molecules are being considered as an attractive candidate owing to their economic viability, reproducibility, and ease of processing. Single-molecule magnets (SMMs) are one such class of molecules exhibiting a bistable ground state and a large magnetic anisotropy, which introduces an energy barrier for spin reversal. ${ }^{1-5}$

The working temperature of the SMM is characterized by its blocking temperature $\left(T_{B}\right)$, below which the molecule shows a magnetic remanence and open hysteresis. With the development of the molecular design, there has been substantial enhancement in the values of the $T_{\mathrm{B}}$. Since an SMM can store information in its spin-state below $T_{B}$, it can be used to realize functional spintronic devices. ${ }^{1,6,7}$

However, an SMM can lose the information stored in its spin-state either due to the dynamic coupling with its environment or via nonthermal pathways such as the quantum tunneling (QT) relaxation process. ${ }^{8-10}$ For the former process, the interaction between the spin and the vibrational modes (phonon) can be the driving force to overcome a potential barrier for spin-flipping. ${ }^{10,11}$ In the latter QT process, two

Received: June 6, 2019

Accepted: November 12, 2019

Published: November 12, 2019 
degenerated spin-states, having opposite spin-directions and placed in the absence of outer magnetic field, tunnel into each other without following the potential curve. ${ }^{8}$ As a result of the QT process, SMMs, in spite of having a large magnetic anisotropy barrier, show vanishing remanence in their magnetic hysteresis curve even at very low temperatures and behave similar to a paramagnetic material. There are continuous efforts to suppress the QT process such as by combination with CNTs ${ }^{9,10,12}$ or adsorption on a $\mathrm{MgO}$ film. ${ }^{13}$ However, further progress requires the atomic-scale characterization of the magnetic behavior of surface-supported SMMs to understand and control the quantum tunneling relaxation of molecular spins.

Here, we demonstrate robust spin-polarization of the double-decker bis(phthalocyaninato)terbium(III) $\left(\mathrm{TbPc}_{2}\right)$ molecules adsorbed on bilayer $\mathrm{Co}$ islands on a $\mathrm{Au}(111)$ substrate using spin-polarized scanning tunneling microscopy (SP-STM). The $\mathrm{TbPc}_{2}$ molecule is a quintessential SMM, ${ }_{11}^{14}$ and its behavior in thin films has attracted wide attention. ${ }^{15-21}$ One of the prerequisites for making devices using SMMs is to adsorb them on a substrate surface. SP-STM is ideally suited for studying SMMs adsorbed on a surface since it can detect both the spin-state and the bonding configuration of the molecules at the same time with submolecular resolution. ${ }^{22-24}$ Our results reveal that molecular spins of a $\mathrm{TbPc}_{2} \mathrm{SMM}$ are polarized not only by the antiferromagnetic coupling to the magnetization of the Co island but also by the intrinsic large magnetic anisotropy energy of the $\mathrm{TbPc}_{2}$ molecule. Stable spin-polarization is inferred from the sizable hysteresis observed in the magnetization curves measured over the $\mathrm{TbPc}_{2}$ molecules attached to the Co islands. In addition, the magnetization curves depict that the spin of the $\mathrm{TbPc}_{2}$ molecule can be switched independently of the Co island magnetization. These results are in contrast to the reports of near-zero remanence for the bulk $\mathrm{TbPc}_{2}$ crystals and $\mathrm{TbPc}_{2}$ molecules on a nonmagnetic substrate. The significant hysteretic opening observed in our study can be attributed to the large magnetic anisotropy barrier of the molecule and the suppression of the quantum tunneling process.

\section{RESULTS AND DISCUSSION}

Co Bilayer Magnetization. Figure 1(a) shows a topographic image of the surface after submonolayer Co deposition onto a $\mathrm{Au}(111)$ surface, resulting in isolated bilayer islands. Some islands contain protruding areas corresponding to the third Co layer. The magnetic properties of these Co islands were probed using a $\mathrm{Pt} / \mathrm{Ir}$ tip after in situ capture of a cobalt cluster from the surface. ${ }^{25}$ These tips were found to be superparamagnetic in nature, and the tip magnetization direction is aligned out-of-plane by application of a small external magnetic field. ${ }^{2 .}$

We first examine the spin-polarized conductance using the single-point scanning tunneling spectroscopy (STS) measurement over Co islands and determine the sample bias voltage to obtain the largest spin contrast. Note that the $\mathrm{d} I / \mathrm{d} V$ contrast is determined by the band structure of the sample at the energy used for the tunneling. Figure 1(b) (lower panel) shows the $\mathrm{d} I / \mathrm{d} V$ spectra measured over a Co island with the parallel $(B=$ $-1 \mathrm{~T})$ and antiparallel $(B=+1 \mathrm{~T})$ relative alignment of the tip and the sample magnetizations. Such relative orientation of magnetization was ensured by applying a saturation magnetic field of $-5 \mathrm{~T}$ prior to the measurement to align the magnetization of the Co island. Since the tip magnetization
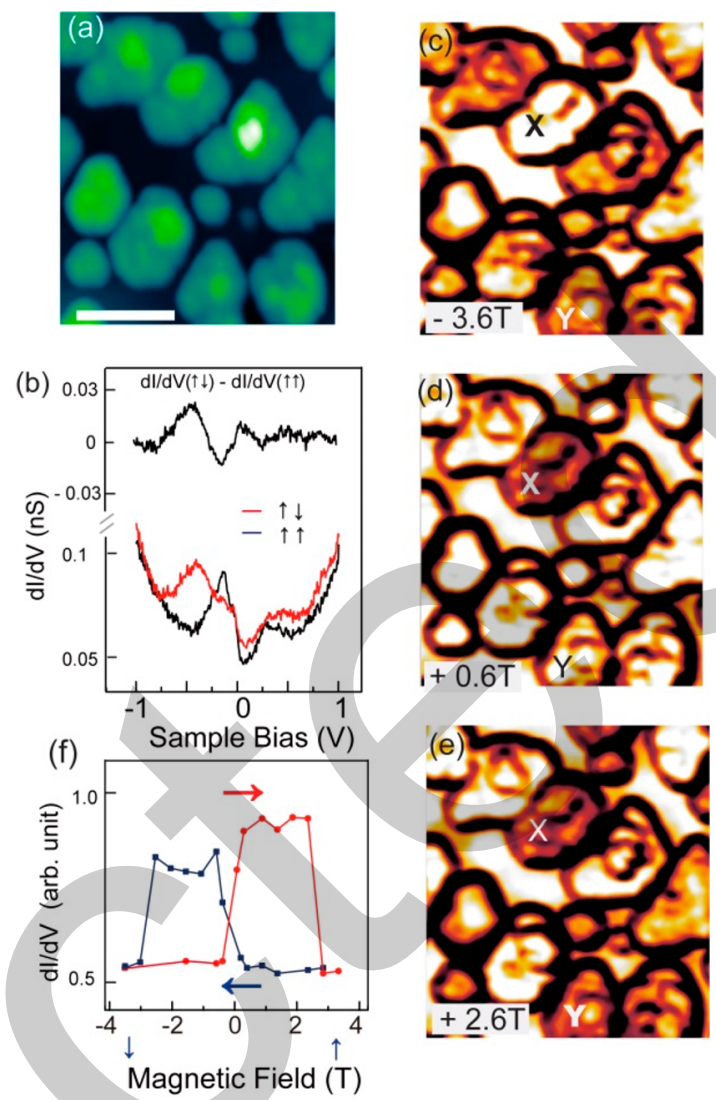

Figure 1. (a) Topographic image $\left(V_{\mathrm{S}}=-600 \mathrm{mV}, I_{\mathrm{t}}=0.2 \mathrm{nA}\right.$, length scale is $10 \mathrm{~nm}$ ). (b) Tunneling spectra over Co bilayer island obtained with $B=+1 \mathrm{~T}$ (red) and $-1 \mathrm{~T}$ (black) (lower panel) and the difference of two spectra (upper panel). All the data were recorded at a temperature of $4.6 \mathrm{~K}$. (c-e) Conductance mapping obtained with $V_{S}=-600 \mathrm{mV}$ with an out-of-plane magnetic field of $-3.6 \mathrm{~T}(\mathrm{c}),+0.6 \mathrm{~T}(\mathrm{~d})$, and $+2.6 \mathrm{~T}(\mathrm{e})$. (f) Representative hysteresis loop for $\mathrm{d} I / \mathrm{d} V\left(V_{\mathrm{S}}=-600 \mathrm{mV}\right)$ obtained at the terrace position of a Co island. Blue and red arrows indicate the sequence of the $B$ change during the measurement.

can be reversed by applying a small magnetic field in the opposite direction, $B=-1 \mathrm{~T}(+1 \mathrm{~T})$ corresponds to a parallel (antiparallel) relative alignment. The spectra reveal an increase of the conductance at $V_{S}=-200 \mathrm{mV}$ for the parallel alignment due to the presence of the Co $\mathrm{d}_{z}{ }^{2}$ surface-state at $\sim-0.2 \mathrm{~V}$. At $V_{\mathrm{S}}=-600 \mathrm{mV}$, the $\mathrm{d} I / \mathrm{d} V$ contrast is reversed as compared to that of $-0.2 \mathrm{~V}$. As a result, a parallel (antiparallel) alignment leads to a dark (bright) contrast on the island indicating a low conductance state at $V_{\mathrm{S}}=-600 \mathrm{mV}$. Since it gives the highest spin contrast, we use this voltage for the spin contrast mapping.

The differential conductivity $(\mathrm{d} I / \mathrm{d} V)$ maps were acquired at $V_{\mathrm{S}}=-600 \mathrm{mV}$ using the conventional lock-in detection with the feedback loop closed. A modulation voltage of $50 \mathrm{mV}$ was superimposed on the tunneling gap voltage. Co islands exhibit spontaneous out-of-plane magnetization, which is manifested in a two-level contrast in the $\mathrm{d} I / \mathrm{d} V$ maps. The two levels of contrast correspond to the parallel or antiparallel alignment of the island magnetization with respect to the out-of-plane component of the tip magnetization.

An external out-of-plane magnetic field was applied perpendicular to the sample surface to change the magnetization state of the Co islands. As shown in Figures 1(c-e), the magnetic field $B$ is sequentially changed from $-3.6 \mathrm{~T}(\mathrm{c})$ to 
$+0.6 \mathrm{~T}(\mathrm{~d})$ and finally to $+2.6 \mathrm{~T}(\mathrm{e})$. All islands appear as either bright or dark owing to their high- or low-conductance state, respectively, at $V_{\mathrm{S}}=-600 \mathrm{mV}$. In Figure 1(c), i.e., at -3.6 $\mathrm{T}$, most of the islands appear as the dark one except that marked as " $X$ " in the figure. At this value of the field, the magnetization of the islands (except the one marked as "X") as well as that of the tip is expected to be aligned along the external field direction. As the field direction is reversed to a small positive value, a conductance contrast reversal occurs over all the islands (Figure 1(d)). This observation is indicative of the flipping of the tip magnetization. As the $B$ field is increased to $+2.6 \mathrm{~T}$, some of the islands flip their magnetization so as to align with the external magnetic field if it exceeds the magnetic coercivity of the Co island. An example can be seen in the Co island marked as "Y" in Figure 1(e), which shows the change from bright (d) to dark (e) as $B$ changes from $+0.6 \mathrm{~T}$ to $+2.6 \mathrm{~T}$. These observations indicate that a parallel (antiparallel) alignment of the tip and sample magnetizations gives dark (bright) contrast over the islands at $V_{S}=-600 \mathrm{mV}$. This is consistent with the results of the singlepoint STS shown in Figure 1(b).

In order to examine the conductance changes in more detail, we measured the $\mathrm{d} I / \mathrm{d} V$ hysteresis loop, where the $\mathrm{d} I / \mathrm{d} V$ signal is plotted as a function of the external magnetic field. A representative hysteresis loop over a Co island is shown in Figure $1(\mathrm{f})$. The $\mathrm{d} I / \mathrm{d} V$ signal over an island at $V_{\mathrm{S}}=-600 \mathrm{mV}$ was measured as the $B$-field is swept starting from $+3 \mathrm{~T}$ along the blue line. After reaching $B=-3.5 \mathrm{~T}$, we return $B$ to its starting value along the red line. Both the blue and the red curves have two symmetric, large changes corresponding to the flipping of the tip $(B \approx 0 \mathrm{~T})$ and the Co island $(B= \pm 3 \mathrm{~T})$ magnetizations. Similar butterfly-shaped hysteresis loops were reported for the Co bilayers islands on $\mathrm{Au}(111)^{25}$ and on $\mathrm{Cu}(111){ }^{26-28}$ Our observations clearly establish that the bilayer $\mathrm{Co}$ islands on $\mathrm{Au}(111)$ exhibit single-domain, magnetically bistable states with large coercivity.

Adsorption Configuration of $\mathrm{TbPc}_{2}$. Figure 2 shows a typical image of the $\mathrm{TbPc}_{2}$ molecules adsorbed on the bilayer Co island on a $\mathrm{Au}(111)$ substrate. $\mathrm{TbPc}_{2}$ molecules were deposited while the substrate temperature was kept close to liquid $\mathrm{N}_{2}$ temperature. Cooling of the substrate was necessary because when the molecules are deposited while the surface of $\mathrm{Co} / \mathrm{Au}(111)$ is at the room temperature, we found that the Co film catalyzes the dissociation of molecules into two separate Pc planes. The height of the $\mathrm{TbPc}_{2}$ molecules from the Co island plane is measured to be $\sim 400 \mathrm{pm}$, and eight lobes are observed at the ligand position of the $\mathrm{TbPc}_{2}$ molecule. Similar height and shape of the molecule were observed in our previous report when the molecule was adsorbed on a $\mathrm{Au}(111)$ surface. $^{21}$ As we calculated in the report, the eight lobes are derived from the two areas of high density-of-states (DOS) flanking the benzene ring of the isoindole. ${ }^{21}$ The height and the shape of the molecule are the hallmarks of the robust double-decker phthalocyanine molecule. Figure 2(a) depicts four Co islands decorated by the eight-lobed $\mathrm{TbPc}_{2}$ molecules which are adsorbed at the edges of the islands and in the middle of the islands (terraces). A few molecules are also seen on the bare gold surface. This can be understood with a model in which the evaporated $\mathrm{TbPc}_{2}$ molecules hop on the inert $\mathrm{Au}(111)$ surface before being trapped at the edges of the Co islands.

We analyze the bonding configuration of the $\mathrm{TbPc}_{2}$ molecule by examining its orientation. The orientation of the
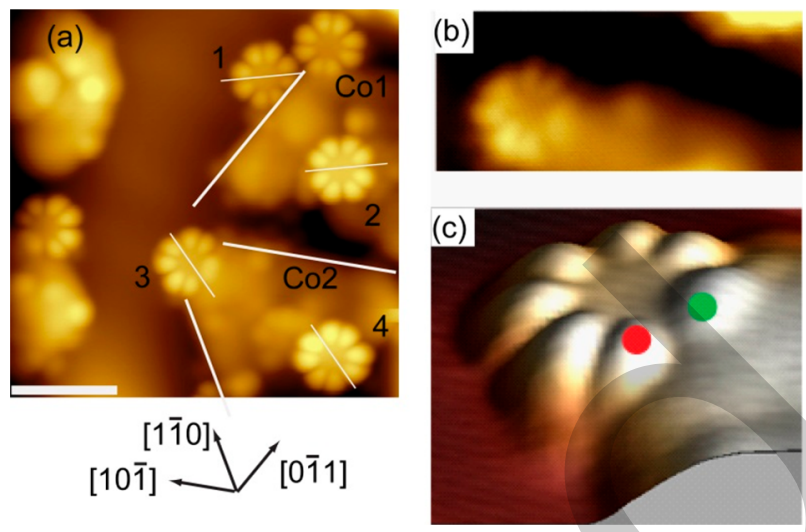

Figure 2. (a) Topographic image of $\mathrm{TbPc}_{2}$ molecules adsorbed on the Co bilayer islands formed on a $\mathrm{Au}(111)$ surface $\left(V_{\mathrm{S}}=-800\right.$ $\mathrm{mV}, I_{\mathrm{t}}=25 \mathrm{pA}$, length scale $\left.=4 \mathrm{~nm}\right)$. Crystallographic directions of the $\mathrm{Au}(111)$ substrate are shown at the bottom. Perimeters of Co islands labeled as $\mathrm{Co} 1$ and $\mathrm{Co} 2$ are depicted by thick white lines, and the symmetry lines of the $\mathrm{TbPc}_{2}$ molecules marked 1 to 4 are shown by thin lines. (b) Zoomed-in topographic image and (c) three-dimensional image of a $\mathrm{TbPc}_{2}$ molecule at the edge of a Co island. The green and red dots indicate the lobes of the Pc ligand attached to the Co island and in-between the Co island and Au substrate, respectively. All the data were recorded at a temperature of $4.6 \mathrm{~K}$.

substrate $\mathrm{Au}(111)$ can be determined from the herringbone structure. The three equivalent directions of $\mathrm{Au}(111)$, which are $[10 \overline{1}],[0 \overline{1} 1]$, and $[1 \overline{1} 0]$ corresponding to the close-packed direction of gold atoms, are depicted at the bottom of Figure 2(a). Clear perimeters of the two Co islands, $\mathrm{Co} 1$ and $\mathrm{Co} 2$, are shown by thick white lines in the figure. It is known that the

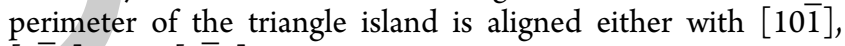
$[0 \overline{1} 1]$, and $[1 \overline{1} 0]$ directions, which is reproduced in Figure $2(a){ }^{25}$ Also, the crystallographic orientation inside the Co bilayer island is aligned with that of the $\mathrm{Au}(111)$ substrate owing to the epitaxial growth of the Co bilayer. ${ }^{25}$ The orientations of the $\mathrm{TbPc}_{2}$ molecules are analyzed using the symmetry lines shown in the figure, which can be seen for the molecules 1 to 4 in Figure 2(a). The lines are drawn in the diagonal direction connecting the middles of the bright lobes. The lines for the molecules 1 and 2 are rotated $15^{\circ}$ from the $[10 \overline{1}]$ direction, and those for 3 and 4 are rotated $15^{\circ}$ from the [1110] direction. Such an orientation of the molecule is exactly the same as what was observed for the $\mathrm{TbPc}_{2}$ molecule adsorbed on a clean $\mathrm{Au}(111)$ surface. $^{21}$ In there, the symmetry line connecting the diagonal isoindoles of the bottom $\mathrm{Pc}$ is aligned with the close-packed direction of $\mathrm{Au}(111)$. This bonding configuration of the $\mathrm{TbPc}_{2}$ molecule is preserved both for the $\mathrm{TbPc}_{2}$ molecule at the edge of the Co island and on the terrace of the Co bilayer island.

Some of the edge molecules show an apparent tilted bonding configuration in which one part of the ligand is above the Au substrate and the other is above the higher Co island. An example is shown in Figure 2(b and c), the latter of which is illustrated in the $3 \mathrm{D}$ image; the green point indicates the position where the molecule attaches to the Co island, and the red point shows a position that is separated from both the Co island and the Au substrate.

Spin-Polarization of $\mathrm{TbPc}_{2}$ Molecule. Figure 3 summarizes the spin-resolved measurements over the surface after depositing a $\mathrm{TbPc}_{2}$ molecule on a $\mathrm{Co} / \mathrm{Au}(111)$ surface. The 
(a)

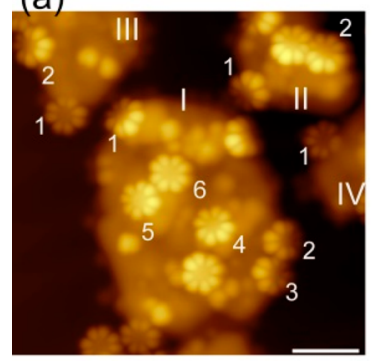

(b)

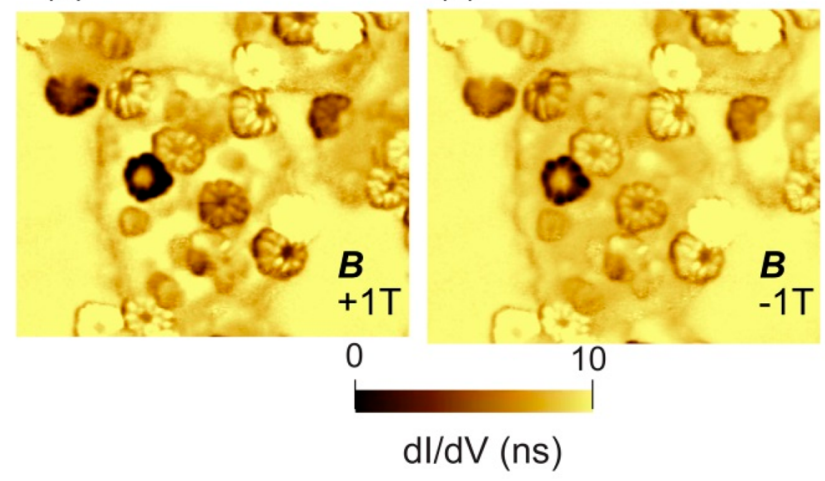

(d)

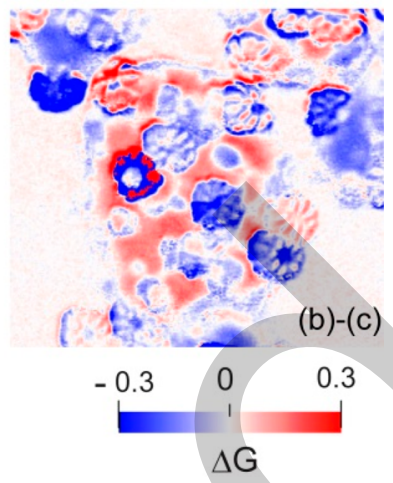

Figure 3. Spin-resolved STM measurements on $\mathrm{TbPc}_{2} / \mathrm{Co} / \mathrm{Au}(111)$. (a) Topographic image of a region showing four Co islands (Co I-IV) together with adsorbed $\mathrm{TbPc}_{2}$ molecules that are numbered for each Co island. $\left(V_{S}=-600 \mathrm{mV}, I_{\mathrm{t}}=25 \mathrm{pA}\right.$, length scale $\left.=4 \mathrm{~nm}\right)$. $(\mathrm{b}, \mathrm{c}) \mathrm{dI} /$ $\mathrm{d} V$ image of the same region shown in (a) obtained with $(\mathrm{b}) B=+1 \mathrm{~T}$ and $(\mathrm{c}) B=-1 \mathrm{~T}\left(V_{\mathrm{S}}=-600 \mathrm{mV}, I_{\mathrm{t}}=50 \mathrm{pA}\right)$. (d) Calculated magnetic asymmetry $\Delta G(+1 \mathrm{~T},-1 \mathrm{~T})$. Color scales for the $\mathrm{d} I / \mathrm{d} V$ image and the magnetic asymmetry are shown in the bottom.

topographic image in Figure 3(a) depicts four bilayer Co islands numbered here from I to IV. These islands are decorated by $\mathrm{TbPc}_{2}$ molecules either at the edges or in the middle of the terrace. The molecules attached to each island are also numbered. Hereafter, we refer to a particular molecule using a label comprising the number assigned to the Co island to which it is attached and that assigned to the molecule itself; for example, the molecule I-1 refers to a molecule that is attached to the Co island labeled I and is assigned the number 1.

Spin-resolved information was obtained by measuring the differential conductance over the surface for the two opposite magnetization directions of the tip. Tip magnetization was aligned along the external field direction by the application of $+1 \mathrm{~T}$ (Figure 3(b)) and $-1 \mathrm{~T}$ (Figure 3(c)), and the $\mathrm{d} I / \mathrm{d} V$ signal was measured at $V_{\mathrm{S}}=-600 \mathrm{mV}$. At these values of the external magnetic fields, the magnetization directions of the Co islands remain fixed owing to their high coercivity. Observation of two level contrasts in $\mathrm{d} I / \mathrm{d} V$ signal over Co islands in Figure 3(a) clearly indicates the spin-sensitivity of the tip. Switching of the tip magnetization direction as the external field direction is changed from $+1 \mathrm{~T}$ (Figure $3(\mathrm{~b})$ ) to $-1 \mathrm{~T}$ (Figure $3(\mathrm{c})$ ) is manifested in a reversal of the $\mathrm{d} I / \mathrm{d} V$ signal contrast over the islands. These results allow us to infer the magnetization direction of the Co islands visible in the studied region. Here, as discussed previously, islands I and II (III and IV) have their magnetizations parallel to that of the tip when the external outof-plane magnetic field is $-1 \mathrm{~T}(+1 \mathrm{~T})$. As a result, we assign islands I and II to be magnetized in down direction $(\downarrow)$, while islands III and IV are assigned to be in the up direction ( $\uparrow)$.

While the spin-dependent changes in the conductance state (Figure $3(\mathrm{~b}$ and $\mathrm{c})$ ) is clearly visible for the Co islands for parallel or antiparallel relative alignment of the tip-island magnetizations, getting a similar visualization over individual molecules requires more careful analysis. In order to reveal such finer details, we calculated magnetic asymmetry ${ }^{29,30}$ or spin contrast mapping, which is shown in Figure 3(d). Here, magnetic asymmetry is defined as

$$
\Delta G\left(B_{1}, B_{2}\right)=\frac{G\left(B_{1}\right)-G\left(B_{2}\right)}{G\left(B_{1}\right)+G\left(B_{2}\right)}
$$

where $G\left(B_{i}\right)$ is the $\mathrm{d} I / \mathrm{d} V$ signal measured at an external magnetic field $B_{i}$. Here in Figure 3(d), the color scale is chosen so that the area of $\Delta G=0$ appears as white, and red and blue colors correspond to a positive and a negative value of $\Delta G$, respectively. Evidently, both the Co islands and the molecules show a finite value of $\Delta G$, while $\Delta G=0$ for the nonmagnetic Au substrate region.

Observation of the finite value of $\Delta G$ over molecules is indicative of the spin-polarized molecular electronic states when attached to the Co islands. However, $\Delta G$ depends sensitively on the bonding configuration and the magnetization direction of the Co island to which the $\mathrm{TbPc}_{2}$ molecule is attached. From the topographic image of Figure 3(a), molecules can be classified in two groups: (1) the edge molecules that are attached to both the Au substrate and Co island and (2) the flat-lying molecules adsorbed on Co island terraces. The edge molecules, when attached to the Co islands magnetized in the down direction $(\downarrow)$, change from the high conductance state to the low conductance state as the external field changes from $+1 \mathrm{~T}$ to $-1 \mathrm{~T}$. Such molecules exhibit a positive value of $\Delta G$ and appear as red in Figure 3(d). Examples of such molecule are I-1, I-2, II-1, and II-2. On the other hand, when the molecule is attached to a Co island magnetized in the up direction $(\uparrow)$, the molecule appears as blue, e.g., III-1 and IV-1. Thus, the spin-contrast over these molecules is reversed as compared to the molecules attached to Co islands magnetized in the down direction $(\downarrow)$.

The flat-lying molecules adsorbed at the terraces of Co islands, e.g., I-3, I-4, and I-5, appear as blue in the spin-contrast map of Figure 3(d), indicative of switching from a low conductance state to a high one as the applied field changes from $+1 \mathrm{~T}$ to $-1 \mathrm{~T}$. Interestingly, for the molecules on the terrace, the spin contrast is opposite to that of the edge molecules attached to the same Co island. It should be noted, however, that the spin-contrast over molecules is extremely sensitive to the small variations in the bonding configuration. As an example, molecules I-2 and I-3 exhibit opposite spincontrasts, even though the changes in the bonding configuration, as ascertained from the topographic image of Figure 3(a), are imperceptible.

The high sensitivity of the spin-contrast to the bonding configuration or adsorption site can be ascribed to a strong molecule-substrate interaction for $\mathrm{TbPc}_{2}$ molecules adsorbed on the Co islands. In order to deduce the dependence of the spin-contrast on the adsorption site, it is instructive to examine the changes in the local density of states over the $\mathrm{TbPc}_{2}$ 
molecule at different locations of the Co islands. Figure 4 illustrates the representative STS results obtained at the lobe
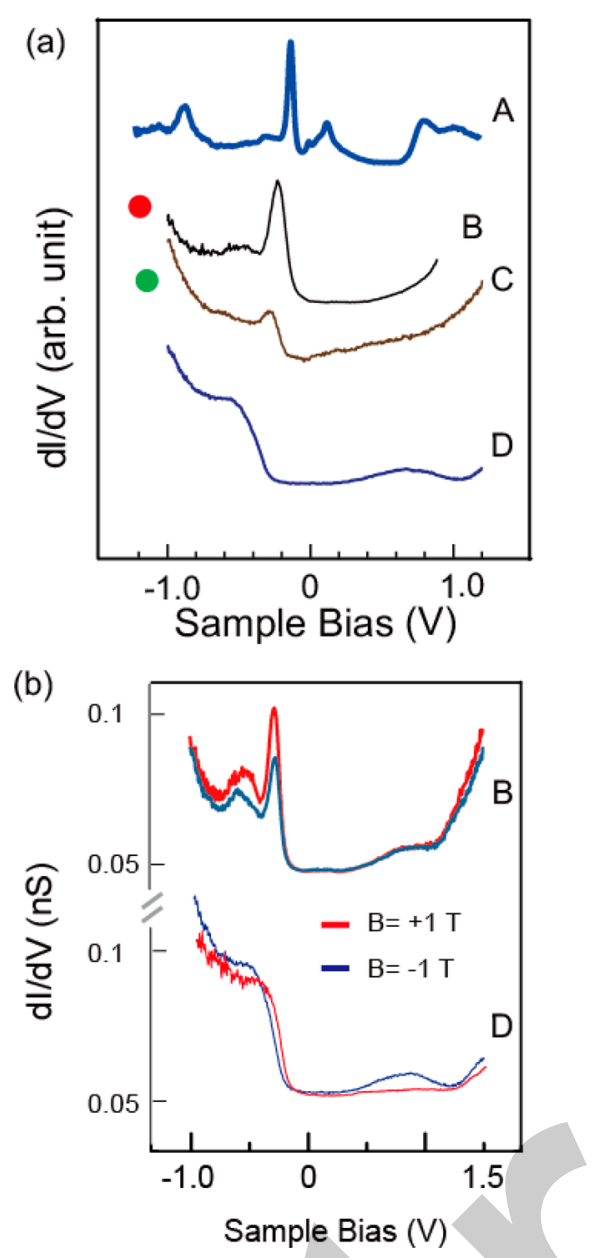

Figure 4. (a) STS spectra of the $\mathrm{TbPc}_{2}$ molecule: (A) reference spectrum for $\mathrm{TbPc}_{2}$ adsorbed on bare $\mathrm{Au}(111)$, (B) obtained at the location of the red dot in Figure 2 (c) for the molecule at the edge of the Co island, (C) obtained at the location of the green dot in Figure 2(c), (D) obtained at the lobe of a molecule adsorbed in the middle of a Co island. (b) Magnetic-field-dependent STS spectra over molecules at the same positions as that for B (upper panel) and $D$ (lower panel) of (a).

positions of the $\mathrm{TbPc}_{2}$ molecule. For comparison, we show a reference spectrum labeled as $\mathrm{A}$ acquired for $\mathrm{TbPc}_{2}$ molecules adsorbed on a pure $\mathrm{Au}(111)$ substrate, as reported previously by our group.

In spectrum $\mathrm{A}$, a sharp peak at the energy of $-0.2 \mathrm{eV}$ arises due to the unscreened final-state effect caused by the weakened interaction between the upper Pc ligand and the $\mathrm{Au}(111)$ substrate. Thus, the molecular nature of the orbital is preserved, as suggested by the narrow peak width, and its energy position depends on the degree of the screening of the final state (ionized state after the tunneling process), which is sensitive to the chemical environment. This observation provides a clue to explain the sensitivity of the spin contrast to the bonding position as stated earlier. The spectra $\mathrm{B}$ and $\mathrm{C}$ correspond to a molecule adsorbed at the Co island edge obtained at the locations marked by red and green dots in Figure 2(c), respectively. Due to the canted bonding configuration, even within the same molecule, the STS feature changes the shape depending on the lobe at which the spectra was measured. The shape of the STS spectrum obtained at the lobe away from the Co island (B) closely resembles that of the spectrum A. However, for the spectrum $\mathrm{C}$ obtained at the lobe directly in contact with the Co island, the sharp peak is observed only weakly followed by a step-like feature at the higher energy region. Such a step-like feature in STS is more prominent for the flat-lying molecules adsorbed on the terrace of the Co island such as molecule 2 in Figure 2(a). A representative spectrum for a flat-lying molecule on a Co island is labeled as D, where only a step-like feature is visible at an energy of $-500 \mathrm{mV}$. This transition from sharp peak to a steplike feature can be accounted for by considering a stronger bonding between a $\mathrm{TbPc}_{2}$ molecule and Co substrate, compared to that for the $\mathrm{Au}$ substrate, which makes the lessshielded sharp peak disappear.

In order to obtain further insights about the spin-polarized electronic states of $\mathrm{TbPc}_{2}$ molecules attached to the Co islands, we measure the change of the $\mathrm{d} I / \mathrm{d} V$ signal as a function of applied magnetic field at $V_{\mathrm{S}}=-600 \mathrm{mV}$. This value of $V_{S}$ was chosen since it gives a clear difference in the local DOS (LDOS) for the two orientations of the tip magnetizations for both the edge molecules and the flat-lying molecules (see Figure 4(b)) and is free from the structural contributions arising due to the $\mathrm{Co}_{z}{ }^{2}$ states located around $V_{\mathrm{S}}$ $\approx-0.2 \mathrm{~V}$, as discussed earlier.

Here we describe the detail of the variation of the STS maps with the change of the magnetic field. Figure 5(a) depicts the topographic image of a selected region magnified from Figure 3(a). The two Co islands, labeled I and III, are magnetized in the directions of $\downarrow$ and $\uparrow$, respectively, and their magnetizations remain unchanged during the magnetic field sweep employed here. The $\mathrm{d} I / \mathrm{d} V$ maps were recorded at different $B$ values, in a stepwise manner, going from $+3.0 \mathrm{~T}$ to $-3.5 \mathrm{~T}$ (forward sweep) and back to $+3.0 \mathrm{~T}$ (backward sweep).

Figure $5(\mathrm{~b}-\mathrm{e})$ show the $\mathrm{d} I / \mathrm{d} V$ mappings obtained during the forward sweep of $B$ for selected $B$ values of $+1,-1,-2$, and $-2.5 \mathrm{~T}$, respectively. In order to examine the variation of the conductance state of the molecules with the magnetic field of $B$, we calculated the magnetic asymmetry between the successive $B$ values. This analysis allows us to assign and monitor the conductance state of the molecules during the field sweep, which can be used to ascertain their magnetization direction. Figure $5(\mathrm{f}-\mathrm{h})$ depicts the calculated magnetic asymmetry corresponding to the values of $\Delta G(+1 \mathrm{~T},-1 \mathrm{~T})$, $\Delta G(-1 \mathrm{~T},-2 \mathrm{~T})$, and $\Delta G(-2 \mathrm{~T},-2.5 \mathrm{~T})$, respectively.

Figure 5(f) further corroborates our analysis of Figure $3(\mathrm{~d})$ that spin contrast appears for molecules both at the edge of the Co islands and on the Co island terraces. As inferred from Figure 5(f), the molecules I-1 and III-1 are the edge molecules, which appear red and blue, respectively. On the other hand, the flat-lying molecules I-5 and I-6 appear as blue in Figure $5(\mathrm{f})$, indicating a reversal of spin contrast over these molecules.

Figure $5(\mathrm{~g})$ reveals the change in the spin contrast over molecules when $B$ is changed from $-1 \mathrm{~T}$ to $-2 \mathrm{~T}$. Very interestingly, the spin contrast over molecules I-5, I-6, and III-1 changes from blue to red, indicating a switching of the conductance state of these molecules. Further changing $B$ from $-2 \mathrm{~T}$ to $-2.5 \mathrm{~T}$ leads to switching of the conductance state of molecule I-1, which now appears as blue in Figure 5(h). It is obvious that only this molecule changes the contrast, indicating a higher magnetic field required to flip the spin of this edge molecule. 

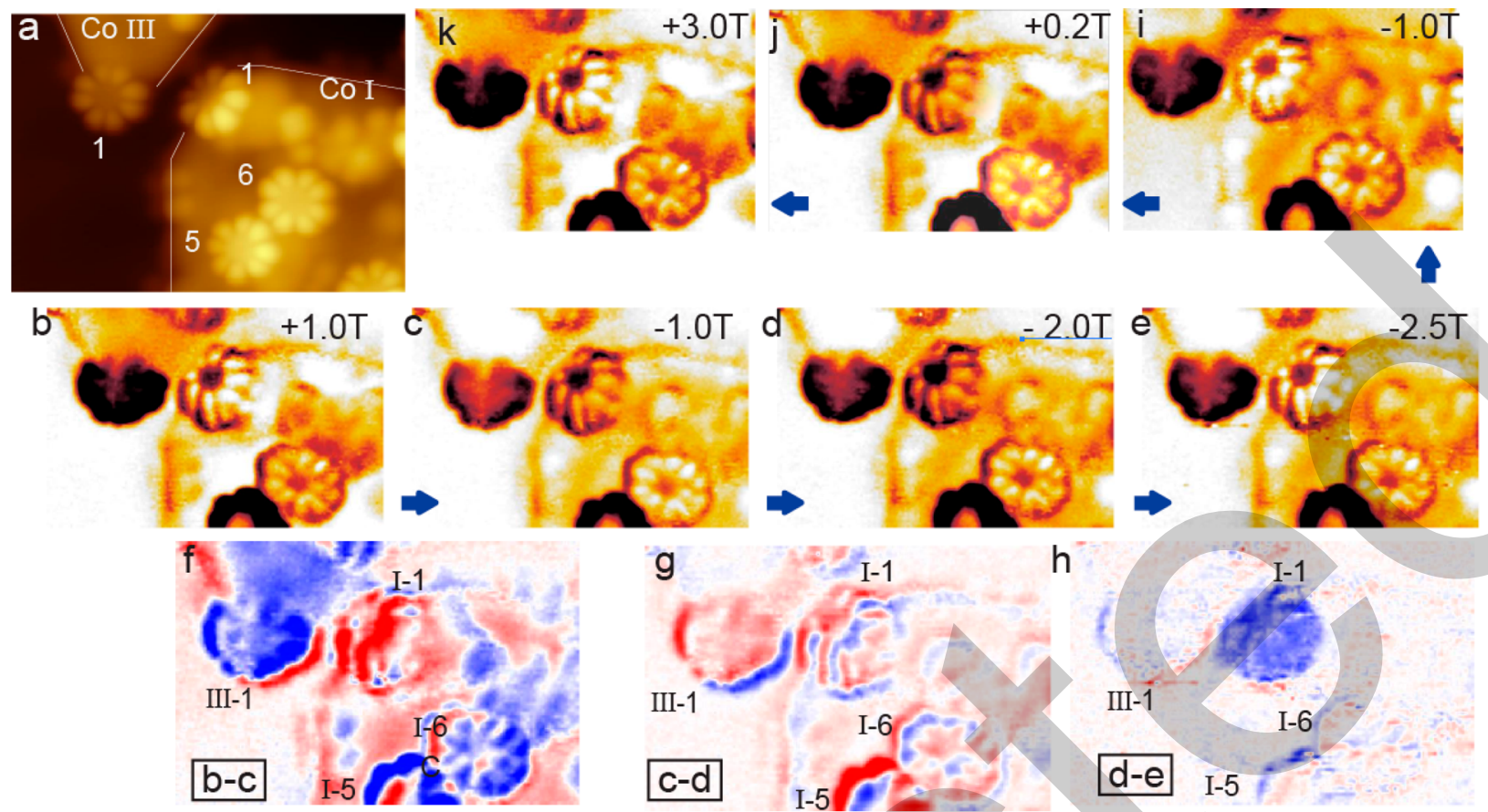

$-1$
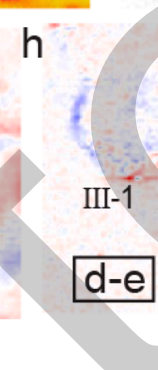

$\mathrm{dl} / \mathrm{dV}$

0 10

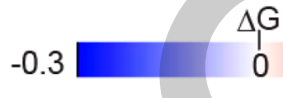

0.3

Figure 5. Variation of the magnetic asymmetry during field sweep. The conductance mapping was obtained at $V_{\mathrm{S}}=-600 \mathrm{mV}$ for the $\mathrm{TbPc}$ molecule adsorbed both at the edges and on the Co island, whose topographic image is shown in (a). Co I and III are identical to that shown in Figure 3. $B$ changed from $+3.0 \mathrm{~T}$ to $-3.5 \mathrm{~T}$ then back to $+3.0 \mathrm{~T}$. The panels of $(\mathrm{b})-(\mathrm{e})$ show the snapshots at the selected $B$ of $+1.0(\mathrm{~b})$, $-1.0(\mathrm{c}),-2.0(\mathrm{~d})$, and $-2.5 \mathrm{~T}(\mathrm{e})$. In addition, the difference mappings of $\Delta G(+1 \mathrm{~T},-0.5 \mathrm{~T}), \Delta G(-0.5 \mathrm{~T},-1 \mathrm{~T})$, and $\Delta G(-1 \mathrm{~T},-2 \mathrm{~T})$ are illustrated in (f), $(\mathrm{g})$, and $(\mathrm{h})$, respectively. After reaching $-3.5 \mathrm{~T}, \mathrm{~B}$ returns to $+3.0 \mathrm{~T}$. Conductance snapshots at $B=-1 \mathrm{~T}(\mathrm{i}),+0.2 \mathrm{~T}(\mathrm{j})$, and $+3.0 \mathrm{~T}(\mathrm{k})$ are illustrated.

As discussed earlier, the conductance state (low or high) is related to the relative magnetization orientation (parallel or antiparallel) between the tip and the sample. Moreover, as long as the direction (sign) of $B$ is unchanged during the sweep, both the tip magnetization direction and that of the Co islands are fixed, within the range of $B$ employed here. Consequently, the observed changes in the conductance state, in Figure $5(\mathrm{~g})$ and (h), over the molecules I-5, I-6, III-1, and I-1 indicate switching of the magnetization direction of the $\mathrm{TbPc}_{2}$ molecules. More importantly, these results clearly demonstrate that $\mathrm{TbPc}_{2}$ molecular spins can be switched independently of the Co island magnetization by the application of the external magnetic field.

Similar magnetization switching is observed again during the backward sweep cycle, where $B$ is swept from -3.5 to $3 \mathrm{~T}$. Figure $5(\mathrm{i}-\mathrm{k})$ show selected $\mathrm{d} I / \mathrm{d} V$ maps at $B$ values of $-1.0 \mathrm{~T}$ $(\mathrm{i}),+0.2 \mathrm{~T}(\mathrm{j})$, and $+3.0 \mathrm{~T}(\mathrm{k})$ obtained during the stepwise backward sweep of $B$. As clearly discernible in Figure 5(i), molecule I-1 exhibits a high conductance state, which is different from the low conductance state seen in the $\mathrm{d} I / \mathrm{d} V$ map of Figure 5(c), acquired at the identical value of $B=-1$ $T$. This observation clearly demonstrates magnetic hysteresis in the $\mathrm{TbPc}_{2}$ molecules attached to the Co islands.

During the backward sweep, as the $B$ value passes $0 \mathrm{~T}$, a flip of the tip magnetization direction leads to reversal of contrast over both the Co islands and the $\mathrm{TbPc}_{2}$ molecules attached to them. In particular, a comparison of Figure 5(i) and $(j)$ distinctly reveals a decrease and an increase of the conductance for I-1 and I-6, respectively. As inferred from the $\mathrm{d} I / d \mathrm{~V}$ map at
$B=+3 \mathrm{~T}$ (Figure $5(\mathrm{k})$ ), further increasing $B$ in the same direction causes magnetization switching of the molecules again and returns their conductance states to the values that were observed before the beginning of the forward sweep (Figure 5(b)).

In Figure 6( $a$ and $b)$, we convert the contrast of the mapping of Figure 5 into a plot in which the value of the $\mathrm{d} I / \mathrm{d} V$ at $V_{\mathrm{S}}=$ $-600 \mathrm{mV} v s$ the magnetic field $B$ is illustrated. Due to the tunneling magnetoresistance (TMR) character of the $\mathrm{d} I / \mathrm{d} V$ curve, this can be regarded as the magnetization curve.

The magnetization curve shown in Figure 6(a) corresponds to the edge molecule (I-1), while that in Figure 6(b) is obtained for the flat-lying molecule (I-5). The blue and red arrows indicate the forward and backward sweep directions of the magnetic field, respectively, between $B=+3.0 \mathrm{~T}$ and -3.5 $\mathrm{T}$. As determined earlier, the Co island I is magnetized in the down direction during the magnetic field sweep. During the forward sweep in Figure 6(a) along the blue arrow, the first significant change occurs at $B=-0.2 \mathrm{~T}$, where the conductance over the molecule drops to a lower value. Another notable change occurs at between $B=-2 \mathrm{~T}$ and $B=$ $-2.5 \mathrm{~T}$, and the conductance over the molecule jumps to a higher value. During the backward sweep along the red arrow direction, the conductance remains almost constant at a high value until it drops to $B=+0.2 \mathrm{~T}$. A further increase in $B$ along the red arrow shows one more jump in the conductance to a high value at $B=+0.5 \mathrm{~T}$.

Similarly, as seen in Figure 6(b), the two successive conductance jumps during the forward sweep along the blue 

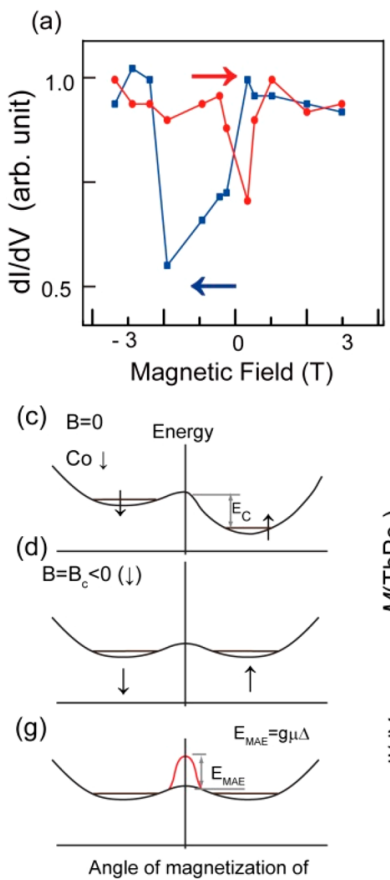

Molecule (b)

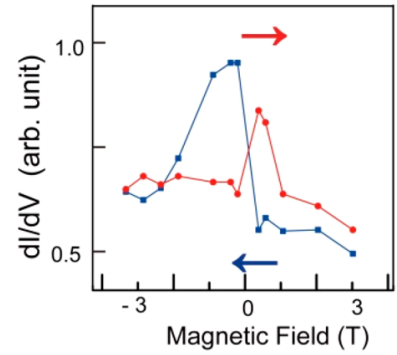

(e)

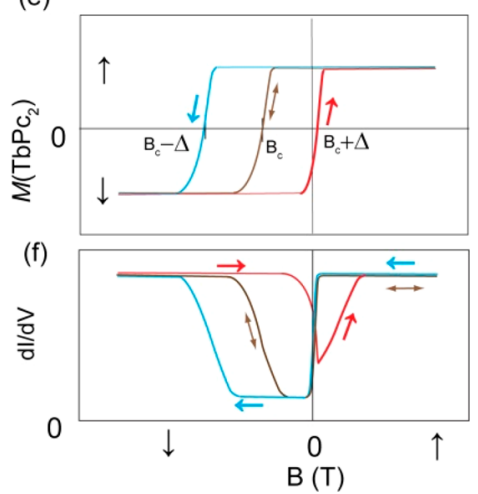

Figure 6. $\mathrm{d} I / \mathrm{d} V$ signal measured as a function of magnetic field for the molecule I-1 (a) and for I-6 (b), which are shown in Figure $5(\mathrm{a})\left(V_{S}=-600 \mathrm{mV}\right)$. Blue and red arrows indicate the sequence of the $B$ change during the measurement. Lines and dots are colored accordingly. Note the magnetization of the Co bilayer is fixed in this range. (c) Double-well energy diagram for the orientation of the molecular spin. Only the ground state doublet corresponding to $J=6, m_{\mathrm{J}}=+6$ and $J=6, m_{\mathrm{J}}=-6$ is shown. Substrate $C o$ is magnetized in the $\downarrow$ direction. $E_{c}$ is the stabilization energy due to the antiferromagnetic exchange coupling between the molecule spin and the Co magnetization. (d) Modified energy levels when $B$ is applied in the $\downarrow$ direction and Zeeman energy becomes equal to $E_{\mathrm{c}}$. (e) Simulated magnetization of the molecule and (f) simulated $\mathrm{d} I / \mathrm{d} V$ hysteresis loop. (g) Energy diagram showing the energy barrier due to the magnetic anisotropy of the $\mathrm{TbPc}_{2}$ molecule.

arrow direction occur near $B=0 \mathrm{~T}$ and between $B=-1.0 \mathrm{~T}$ and $B=-2.0 \mathrm{~T}$, while during the backward sweep the distinct jumps occur at $B=+0.2 \mathrm{~T}$ and $B=+1.0 \mathrm{~T}$. The magnetic field for the second jump during the forward sweep along the blue line is smaller than that observed for the edge molecule in Figure 6(a). This is commonly observed for the groups of the edge molecules and the flat-lying molecules on the Co terrace. The magnetization curves shown in Figure 6(a and b) over the edge molecule and the flat-lying molecule closely resemble the butterfly hysteresis loop obtained for the Co islands (Figure 1(f)). Moreover, both the curves exhibit similar behavior, except for the exact value of the second conductance jump during the field sweep in any direction, if we flip one of the curves vertically. Following the argument for switching of the Co island magnetization along $B$ at a value larger than its coercivity, it is reasonable to assume that the molecule magnetization is aligned along the applied field direction at the terminal values of $B=-3.5 \mathrm{~T}$ and $B=+3.0 \mathrm{~T}$. Thus, our analysis reveals that parallel alignment of the tip and molecule magnetizations yields a high conductance state for the edge molecules (Figure 6(a)) and a low conductance state for the flat-lying molecules (Figure 6(b)). Consequently, even though the measured magnetization curves are vertically flipped, the

magnetization directions of both the edge molecule and the flat-lying molecules at the terminal field values are identical i.e., along the applied field direction. Observed reversal of the sign of magnetic asymmetry for the edge molecule, as compared to that of the flat-lying molecule, can be ascribed to the difference in the molecule DOS, which originates from the different molecule-Co island interaction for the two cases, as discussed previously using point spectroscopy. As evident from point spectra shown in Figure 4(b), the sign of magnetic asymmetry is the same for both the edge molecule (spectra B) and the flatlying molecule (spectra D) attached to same Co island when $V_{\mathrm{S}} \approx-0.3 \mathrm{~V}$, while it becomes opposite when $V_{\mathrm{S}} \approx-0.6 \mathrm{~V}$. To summarize, the observed vertical flip of magnetization curves of Figure $6(a$ and $b)$ is driven by the DOS difference of the molecules with different bonding configurations, while the magnetization of the molecules is identical for the two cases.

We would like to stress again, although the curve shape is similar, the edge molecule shows the large opening in the hysteresis curve.

Here we discuss the origin of the magnetization curves obtained on $\mathrm{TbPc}_{2}$ molecules. Although complicated, it can be rationalized by considering the following two issues: (1) the coupling of the molecular magnetization to that of the ferromagnetic Co island and (2) the large magnetic anisotropy energy of the molecule. The coupling between the ferromagnetic substrate and the adsorbed molecule has been studied in previous reports. ${ }^{30,32-34}$ For the $\mathrm{TbPc}_{2}$ molecule adsorbed on Co covered $\operatorname{Ir}(111)$ studied using SP-STM, Schwöbel et al. showed spin-splitting of the molecular orbital due to the direct magnetic coupling between the Co island and Pc rings. ${ }^{32}$ Using the XMCD method, which allows the detection of element-sensitive magnetization, Rizzini et al. reported that for the $\mathrm{TbPc}_{2}$ molecules adsorbed on the $\mathrm{Ni}$ surface the $\mathrm{Tb}$ spin is antiferromagnetically coupled with the $\mathrm{Ni}$ magnetization through the ligand-mediated superexchange. ${ }^{33}$ Using an element-resolved hysteresis loop, it was shown that the spin state of the $\mathrm{Tb}$ atom is determined by a competition between the antiferromagnetic coupling with the ferromagnetic substrate and the Zeeman interaction due to an external magnetic field.

On the basis of the insight obtained in the report by Rizzini et al., we illustrate the energy diagram in Figure 6(c). However, since the tunneling magnetoresistance measured in our work is not element specific, we cannot specify whether the observed spin contrast over the molecules arises from the $\mathrm{Tb}$ spin or the ligand spin. We refer to the origin of the spin contrast as the molecule spin. In this plot, the $x$-axis corresponds to the orientation of the magnetization of the molecule spin and the $y$-axis is the energy. We assume here the Co substrate is magnetized in the $\downarrow$ direction. In the absence of the external magnetic field, molecular spin-state in the $\uparrow$ direction is stabilized by the energy $E_{c}$ due to the antiferromagnetic exchange with the Co island. With the application of $B$ in the $\downarrow$ direction, the molecule spin-state in the $\downarrow$ direction is stabilized by the Zeeman energy. When $B$ is increased such that the Zeeman energy overcomes the $E_{\mathcal{c}}$, the molecule spin-state in the $\downarrow$ direction becomes energetically favored, leading to the flipping of the molecular spin. The threshold $B$ at which the molecular spin reorients along the applied field direction is expressed as $B_{\mathrm{c}}$ (Figure $6(\mathrm{~d})$ ). For the application of $B$ in the $\uparrow$ direction, no such spin-flip occurs since both the antiferromagnetic coupling with the Co island and Zeeman interaction favor the molecule to be in the $\uparrow$ spin-state. 
The simulated magnetization curve of the molecule, based on the above model, is illustrated in Figure 6(e) by the brown line. During the forward sweep of the external magnetic field from positive value to negative value, the spin-state of the molecule reorients from $\uparrow$ to $\downarrow$ when the field is reduced below $B_{\mathrm{c}}$. Taking into account the magnetization switching of the tip, as observed in our experiment, and assuming a high conductance for the parallel configuration $(\uparrow \uparrow)$ of the relative tip and the molecule magnetizations, the experimental magnetization curve should appear like the brown curve shown in Figure 6(f). The resulting curve indeed shows two conductance jumps, as observed during the forward sweep of $B$ in Figure 6(a). However, during the backward sweep of the field from negative to positive $B$ values, the model predicts a reversible magnetization curve. Hence this model alone cannot account for the observed hysteresis in the magnetization curves shown in Figure 6(a).

Further refinement of the above model by incorporating the magnetic anisotropy of the $\mathrm{TbPc}_{2}$ and assuming a suppression of the quantum tunneling process explains all the features of the magnetization curve obtained in our experiment. $\mathrm{TbPc}_{2}$ is a single-molecule magnet and possesses a bistable ground state characterized by the large magnetic moment $\left(J=6, m_{\mathrm{J}}=+6\right.$ and -6). Their large magnetic anisotropy is derived from a highly axial ligand field produced by a square-antiprismatic coordination geometry. ${ }^{35}$ This large magnetic anisotropy generates a huge energy barrier of several hundreds of Kelvin ${ }^{36}$ between the ground state and the first excited state, thereby leading to a slow relaxation of magnetization and magnetic hysteresis. However, the Hamiltonian describing the ligand field also contains off-diagonal terms which induce a mixing of the $m_{\mathrm{J}}=+6$ and $m_{\mathrm{J}}=-6$ states, giving an avoided level crossing with an energy barrier of $\sim 1 \mu \mathrm{K}$, effectively causing the QT of magnetization. As a result, only small openings in the hysteresis curve were often observed in the experiments for the bulk $\mathrm{TbPc}_{2}$ and the ones adsorbed on a nonmagnetic substrate primarily due to the QT process, which renders the large magnetic anisotropy of the molecule ineffective. If we assume that the QT process is suppressed and include an energy barrier due to the magnetic anisotropy of the $\mathrm{TbPc}_{2}$ molecule, the potential curve is modified as shown by the red line in Figure $6(\mathrm{~g})$. In this case, both the $\mathrm{TbPc}_{2}$ magnetization (Figure 6(e)) and the experimental magnetization curve (Figure 6(f)) should show a hysteresis with certain opening. Assuming the energy barrier as $\Delta$, the curves for the forward and backward sweep of $B$ are shifted by $\Delta$ toward negative and positive directions, respectively. This is depicted using blue (forward sweep) and red lines (backward sweep) in Figure 6(e and $\mathrm{f}$ ). Evidently, the simulated experimental magnetization curve of Figure 6(f) now reproduces all the features observed in Figure 6(a). Similar analysis predicts that for the edge molecules attached to the Co island magnetized in the $\uparrow$ direction, e.g., molecule II-1, the experimental magnetization curve should be horizontally flipped about $B=0 \mathrm{~T}$. Furthermore, considering the different DOS for the edge and the flat-lying molecules, all the spin-contrast changes observed in Figures 3, 5, and 6 can be interpreted.

There is sound grounds to rationalize our assumption that the QT process is suppressed in the present study. Katoh and co-workers recently showed that by tuning the stacking of $\mathrm{TbPc}_{2}$ molecules such that the $\mathrm{Tb}-\mathrm{Tb}$ direction of neighboring molecules is aligned with the easy axis of the molecule, there appears a substantial suppression of the QT process. $^{37-39}$ The suppression of the QT process was attributed to the ferromagnetic dipole interaction between the spins of neighboring molecules. These results are wellsupported by a theoretical simulation that reproduces the suppression of QT. ${ }^{40}$ A similar mechanism, where a coupling to the ferromagnetic Co island can suppress the QT process, may be responsible for the observation of stable spin-contrast over molecules in our study. A more precise description, however, requires theoretical calculations to better understand the coupling between the molecular spins and the ferromagnetic Co support. The atomically resolved results presented here will provide valuable inputs for further studies in this direction.

Finally we discuss the larger gap opening for the edge molecules than that for the flat-lying molecules. The asymmetric STM images of metal phthalocyanine molecules at the step edges were observed in previous reports. Zhang and co-workers studied the adsorption configuration of the FePc molecule at the monatomic step edge of the $\mathrm{Au}(111)$ surface comparing the STM images and the theoretical calculations including the search of the most stable bonding configuration. $^{41}$ They found that the canted FePc bonding at the step edge, in which the central metal atom of $\mathrm{Fe}$ is attached to the $\mathrm{Au}$ atom of the upper terrace of the step edge, gives the most stable bonding configuration. For this configuration, they claimed that the heights of the lower lobes and the higher lobes from the lower and higher $\mathrm{Au}$ terrace increase by $\sim 1.70$ and $\sim 0.50 \AA$, respectively, compared with the flat-lying molecule. This makes the FePc lobes lifted from the substrate. We believe a similar situation occurs for the lower Pc ligand of the $\mathrm{TbPc}_{2}$ molecule at the step edge of the Co island on $\mathrm{Au}(111)$.

As discussed already, the coupling between the spin and the environment phonon plays a crucial role for the spin flipping of SMM. ${ }^{10,11}$ With the formation of the substrate-molecule bonding, there appear the vibrational modes, such as the hindered-translational mode and the hindered-rotational modes, in the energy region between 1 and $10 \mathrm{meV}$. The low-energy vibrational modes of molecules on metal surfaces were studied well for small molecules. For the $\mathrm{CO}$ molecule adsorbed on $\operatorname{Pd}(110)$ and $\mathrm{Cu}(110)$ surfaces, it is shown that the former mode is responsible for the tunneling-electroninduced surface hopping (the energy for $\mathrm{Cu}(110)$ is $\sim 4.2$ $\mathrm{meV}){ }^{42}$ The change of the energies of these modes at the step edges compared to the case at the terrace was also reported previously. ${ }^{4,44}$

For the $\mathrm{TbPc}_{2}$ molecule at the step edge, it is speculated that, by the lifting of the lobes from the substrate, the coupling of the spin and the low-energy vibrational modes is suppressed if compared with the case of the flat-lying molecule. This change should contribute to the wide opening of the hysteresis curve for the step-edge molecules. This also suggests that spin stability of the SMM molecule might be engineered by controlling the bonding sites on the substrate.

\section{CONCLUSIONS}

In conclusion, we demonstrate robust spin-polarization of $\mathrm{TbPc}_{2}$ molecules adsorbed on the bilayer Co islands grown on $\mathrm{Au}(111)$ using spin-polarized scanning tunneling microscopy. Our results reveal that molecular spins of $\mathrm{TbPc}_{2} \mathrm{SMM}$ are antiferromagnetically coupled to the magnetization of the Co island, irrespective of the bonding site of the molecule. Both the edge molecules and the flat-lying molecule exhibit stable spin directions, opposite to the magnetization direction of the 
Co island to which they are attached. More importantly, the $\mathrm{TbPc}_{2}$ spins can be switched independently of the Co island by the application of an external magnetic field. The magnetic asymmetry for the edge molecules and the flat-lying molecule is opposite due to differences in the molecule DOS which originates from the different molecule-Co island interaction for the two bonding configurations. Stable spin-polarization is inferred from the substantial hysteresis observed in magnetization curves measured over the $\mathrm{TbPc}_{2}$ molecules. In contrast to the reports of near-zero remanence for the bulk $\mathrm{TbPc}_{2}$ crystals and $\mathrm{TbPc}_{2}$ molecules on a non-magnetic substrate, our observation of significant hysteretic opening in the magnetization curve suggests a suppression of the QT process.

The SMM class of molecules have potential technological applications, both as an element of magnetic storage devices and for implementing qubits for quantum computers. While for the former application a high anisotropy barrier and suppression of QT is desired, the latter application requires QT processes for the read-out and manipulation of the qubits. Thus, developing a viable means to tune the $\mathrm{QT}$ processes in a controlled manner can enable us to use the same material to realize both applications. We anticipate that our approach of using the substrate to control and enhance the properties of SMM will accelerate further research toward realization of SMM-based functional spintronic devices.

\section{METHODS}

Experimental Details. Experiments were performed at $4.6 \mathrm{~K}$ in a commercial low-temperature STM from Unisoku with a base pressure of $10^{-8} \mathrm{~Pa}$. Magnetic fields up to $B= \pm 5 \mathrm{~T}$ were applied normal to the sample surface, which is the easy magnetization direction of the bilayer $\mathrm{Co}$ islands on $\mathrm{Au}(111)$. The $\mathrm{Au}(111)$ substrate was prepared by repeated cycles of $\mathrm{Ar}^{+}$ion sputtering followed by annealing. Submonolayer Co was deposited by electron bombardment heating of a Co rod.

The use of the Co bilayer as the substrate has several benefits. First, Co islands are well-characterized using several different techniques ${ }^{25}$ and even used as a standard sample to confirm the spin-polarization of the STM tip. Moreover, simultaneous spin-resolved measurement over both the Co bilayer and the magnetic molecules makes the SPSTM observation reliable. More importantly, it has been shown that the Co bilayer is out-of-plane magnetized and the spin direction of the $\mathrm{TbPc}_{2}$ molecule is aligned perpendicular to the Pc plane. Thus, if the molecule adsorbs in a flat-lying manner, both spins are aligned and we can expect interesting interactions.

Samples were then quickly transferred to the STM precooled at 4.6 $\mathrm{K}$. We keep the $\mathrm{Co} / \mathrm{Au}(111)$ sample at low temperature throughout the experiments to avoid the intermixing of the two with a thermal activation. The molecules of $\mathrm{TbPc}_{2}$ were deposited onto the surface of $\mathrm{Co} / \mathrm{Au}(111)$, which was also kept at low temperature to avoid the dissociation of the molecules.

A low-temperature, ultra-high-vacuum, high magnetic field STM (USM 1300, Unisoku, Japan) was employed. The spin-polarized tip was prepared by in situ capture of a Co cluster from the surface. A PtIr tip was cleaned by $\mathrm{Ar}^{+}$ion sputtering. In order to pick the Co atom/cluster from the surface, first the tip was located above a Co island and then approached toward the island with the feedback loop open. A small voltage pulse, which is given while approaching the tip with feedback off, leads to transfer of Co from the substrate to the tip. A similar vertical manipulation technique has been previously employed by Loth et al. to make reproducible spin-polarized STM tips. ${ }^{45}$ This procedure yields superparamagnetic tips whose magnetization direction can be easily aligned along the desired direction by applying a small external magnetic field of magnitude $50 \mathrm{mT} .^{25}$ The differential conductance $(\mathrm{d} I / \mathrm{d} V)$ mapping was done in constantcurrent mode using standard lock-in detection with the feedback loop closed. A modulation voltage of $50 \mathrm{mV}$ and frequency of $941 \mathrm{~Hz}$ were superimposed to the tunneling voltage. The scan speed during $\mathrm{d} I / \mathrm{d} V$ mapping was kept at $10 \mathrm{~nm} / \mathrm{s}$.

One noteworthy aspect of our study is that the tunneling current used to obtain spin-resolved conductance maps is $\sim 50 \mathrm{pA}$, indicating high spin-sensitivity of the tips. Such low value of tunneling current is an order or two magnitude less than that used in earlier spin-resolved studies reported over molecules. ${ }^{30,32-34}$ Since a small value of tunneling current means small tip-sample interaction, such nonperturbative imaging condition is crucial to preserve the magnetization direction and the electronic structure of the tip over a long period of time necessary to obtain data such as that reported in our paper.

\section{AUTHOR INFORMATION}

\section{Corresponding Authors}

*E-mail: puneet@cusb.ac.in.

*E-mail: yamasita@agnus.chem.tohoku.ac.jp.

*E-mail: tadahiro.komeda.al@tohoku.ac.jp.

\section{ORCID}

Puneet Mishra: 0000-0003-2890-432X

Keiichi Katoh: 0000-0002-0140-8222

Tadahiro Komeda: 0000-0003-2342-8184

\section{Notes}

The authors declare no competing financial interest.

\section{ACKNOWLEDGMENTS}

This study was supported in part by Grant-in-Aid for Scientific Research (S) (No.19H05621 for T.K.), Scientific Research (B) (No. 16H04191 for T.K.), and Exploratory Research (No. 17K19047 for T.K.) and partly supported by a Grant-in-Aid for Scientific Research (C) (Grant No. 15K05467) from MEXT, Japan Society for the Promotion of Science (JSPS). This work was also partially supported by MEXT, JST, Core Research for Evolutional Science and Technology (No. JRMJCR12L3). In addition, M. Yamashita thanks the 111 project (China) (No. B18030) for support. P.M. thanks UGC, India, for financial support under the Faculty Recharge Programme (FRP).

\section{REFERENCES}

(1) Christou, G.; Gatteschi, D.; Hendrickson, D. N.; Sessoli, R. Single-Molecule Magnets. MRS Bull. 2000, 25, 66-71.

(2) Gatteschi, D.; Sessoli, R.; Cornia, A. Single-Molecule Magnets Based on Iron(III) Oxo Clusters. Chem. Commun. 2000, 9, 725-732.

(3) Gatteschi, D.; Sessoli, R. Quantum Tunneling of Magnetization and Related Phenomena in Molecular Materials. Angew. Chem., Int. Ed. 2003, 42, 268-297.

(4) Mannini, M.; Pineider, F.; Sainctavit, P.; Danieli, C.; Otero, E.; Sciancalepore, C.; Talarico, A. M.; Arrio, M. A.; Cornia, A.; Gatteschi, D.; Sessoli, R. Magnetic Memory of a Single-Molecule Quantum Magnet Wired to a Gold Surface. Nat. Mater. 2009, 8, 194-197.

(5) Mannini, M.; Bonacchi, D.; Zobbi, L.; Piras, F. M.; Speets, E. A.; Caneschi, A.; Cornia, A.; Magnani, A.; Ravoo, B. J.; Reinhoudt, D. N.; Sessoli, R.; Gatteschi, D. Advances in Single-Molecule Magnet Surface Patterning through Microcontact Printing. Nano Lett. 2005, 5, 14351438.

(6) Wernsdorfer, W.; Aliaga-Alcalde, N.; Hendrickson, D. N.; Christou, G. Exchange-Biased Quantum Tunnelling in a Supramolecular Dimer of Single-Molecule Magnets. Nature 2002, 416, 406-409.

(7) Gatteschi, D.; Sessoli, R.; Villain, J. Molecular Nanomagnets; Oxford Univ. Press: New York, 2007.

(8) Ishikawa, N.; Sugita, M.; Wernsdorfer, W. Quantum Tunneling of Magnetization in Lanthanide Single-Molecule Magnets: Bis(Phthalocyaninato)Terbium and Bis(Phthalocyaninato)Dysprosium Anions. Angew. Chem., Int. Ed. 2005, 44, 2931-2935. 
(9) Ganzhorn, M.; Klyatskaya, S.; Ruben, M.; Wernsdorfer, W. Quantum Einstein-de Haas Effect. Nat. Commun. 2016, 7, 11443.

(10) Urdampilleta, M.; Klyatskaya, S.; Cleuziou, J. P.; Ruben, M.; Wernsdorfer, W. Supramolecular Spin Valves. Nat. Mater. 2011, 10, 502-506.

(11) Lunghi, A.; Totti, F.; Sessoli, R.; Sanvito, S. The Role of Anharmonic Phonons in Under-Barrier Spin Relaxation of Single Molecule Magnets. Nat. Commun. 2017, 8, 14620.

(12) Ganzhorn, M.; Klyatskaya, S.; Ruben, M.; Wernsdorfer, W. Strong Spin-Phonon Coupling between a Single-Molecule Magnet and a Carbon Nanotube Nanoelectromechanical System. Nat. Nanotechnol. 2013, 8, 165-169.

(13) Wäckerlin, C.; Donati, F.; Singha, A.; Baltic, R.; Rusponi, S.; Diller, K.; Patthey, F.; Pivetta, M.; Lan, Y.; Klyatskaya, S.; Ruben, M.; Brune, H.; Dreiser, J. Giant Hysteresis of Single-Molecule Magnets Adsorbed on a Nonmagnetic Insulator. Adv. Mater. 2016, 28, 51955199.

(14) Ishikawa, N.; Sugita, M.; Ishikawa, T.; Koshihara, S.; Kaizu, Y. Lanthanide Double-Decker Complexes Functioning as Magnets at the Single-Molecular Level. J. Am. Chem. Soc. 2003, 125, 8694-8695.

(15) Vitali, L.; Fabris, S.; Conte, A. M.; Brink, S.; Ruben, M.; Baroni, S.; Kern, K. Electronic Structure of Surface-Supported Bis(Phthalocyaninato) Terbium(III) Single Molecular Magnets. Nano Lett. 2008, 8, 3364-3368.

(16) Moreno Pineda, E.; Komeda, T.; Katoh, K.; Yamashita, M.; Ruben, M. Surface Confinement of $\mathrm{TbPc}_{2}-\mathrm{SMMs}$ : Structural, Electronic and Magnetic Properties. Dalton Trans 2016, 45, 18417-18433.

(17) Katoh, K.; Komeda, T.; Yamashita, M. The Frontier of Molecular Spintronics Based on Multiple-Decker Phthalocyaninato TbIII Single-Molecule Magnets. Chem. Rec. 2016, 16, 987-1016.

(18) Komeda, T. Spins of Adsorbed Molecules Investigated by the Detection of Kondo Resonance. Surf. Sci. 2014, 630, 343-355.

(19) Katoh, K.; Isshiki, H.; Komeda, T.; Yamashita, M. MultipleDecker Phthalocyaninato $\mathrm{Tb}$ (III) Single-Molecule Magnets and Y(III) Complexes for Next Generation Devices. Coord. Chem. Rev. 2011, 255, 2124-2148.

(20) Katoh, K.; Yoshida, Y.; Yamashita, M.; Miyasaka, H.; Breedlove, B. K.; Kajiwara, T.; Takaishi, S.; Ishikawa, N.; Isshiki, H.; Zhang, Y. F.; Komeda, T.; Yamagishi, M.; Takeya, J. Direct Observation of Lanthanide(III)-Phthalocyanine Molecules on $\mathrm{Au}(111)$ by Using Scanning Tunneling Microscopy and Scanning Tunneling Spectroscopy and Thin-Film Field-Effect Transistor Properties of $\mathrm{Tb}$ (III)- and Dy(III)-Phthalocyanine Molecules. J. Am. Chem. Soc. 2009, 131, 9967-9976.

(21) Komeda, T.; Isshiki, H.; Liu, J.; Zhang, Y.-F.; Lorente, N. s.; Katoh, K.; Breedlove, B. K.; Yamashita, M. Observation and Electric Current Control of a Local Spin in a Single-Molecule Magnet. Nat. Commun. 2011, 2, 217.

(22) Wiesendanger, R. Spin Mapping at the Nanoscale and Atomic Scale. Rev. Mod. Phys. 2009, 81, 1495-1550.

(23) Wiesendanger, R.; G̈ntherodt, H. J.; G̈ntherodt, G.; Gambino, R. J.; Ruf, R. Observation of Vacuum Tunneling of Spin-Polarized Electrons with the Scanning Tunneling Microscope. Phys. Rev. Lett. 1990, 65, 247-250.

(24) Bogani, L.; Wernsdorfer, W. Molecular Spintronics Using Single-Molecule Magnets. Nat. Mater. 2008, 7, 179-186.

(25) Mishra, P.; Qi, Z. K.; Oka, H.; Nakamura, K.; Komeda, T. Spatially Resolved Magnetic Anisotropy of Cobalt Nanostructures on the $\mathrm{Au}(111)$ Surface. Nano Lett. 2017, 17, 5843-5847.

(26) Rodary, G.; Wedekind, S.; Sander, D.; Kirschner, J. Magnetic Hysteresis Loop of Single Co Nano-Islands. Jpn. J. Appl. Phys. 2008, 47, 9013-9015.

(27) Oka, H.; Tao, K.; Wedekind, S.; Rodary, G.; Stepanyuk, V. S.; Sander, D.; Kirschner, J. Spatially Modulated Tunnel Magnetoresistance on the Nanoscale. Phys. Rev. Lett. 2011, 107, 187201.

(28) Oka, H.; Ignatiev, P. A.; Wedekind, S.; Rodary, G.; Niebergall, L.; Stepanyuk, V. S.; Sander, D.; Kirschner, J. Spin-Dependent
Quantum Interference within a Single Magnetic Nanostructure. Science 2010, 327, 843-846.

(29) Oka, H.; Brovko, O. O.; Corbetta, M.; Stepanyuk, V. S.; Sander, D.; Kirschner, J. Spin-Polarized Quantum Confinement in Nanostructures: Scanning Tunneling Microscopy. Rev. Mod. Phys. 2014, 86, $1127-1168$

(30) Brede, J.; Wiesendanger, R. Spin-Resolved Characterization of Single Cobalt Phthalocyanine Molecules on a Ferromagnetic Support. Phys. Rev. B: Condens. Matter Mater. Phys. 2012, 86, 184423.

(31) Komeda, T.; Isshiki, H.; Liu, J.; Katoh, K.; Yamashita, M. Variation of Kondo Temperature Induced by Molecule-Substrate Decoupling in Film Formation of Bis(Phthalocyaninato)Terbium(III) Molecules on $\mathrm{Au}(111)$. ACS Nano 2014, 8, 4866-4875.

(32) Schwöbel, J.; Fu, Y.; Brede, J.; Dilullo, A.; Hoffmann, G.; Klyatskaya, S.; Ruben, M.; Wiesendanger, R. Real-Space Observation of Spin-Split Molecular Orbitals of Adsorbed Single-Molecule Magnets. Nat. Commun. 2012, 3, 953.

(33) Lodi Rizzini, A.; Krull, C.; Balashov, T.; Kavich, J. J.; Mugarza, A.; Miedema, P. S.; Thakur, P. K.; Sessi, V.; Klyatskaya, S.; Ruben, M.; Stepanow, S.; Gambardella, P. Coupling Single Molecule Magnets to Ferromagnetic Substrates. Phys. Rev. Lett. 2011, 107, 177205.

(34) Iacovita, C.; Rastei, M. V.; Heinrich, B. W.; Brumme, T.; Kortus, J.; Limot, L.; Bucher, J. P. Visualizing the Spin of Individual Cobalt-Phthalocyanine Molecules. Phys. Rev. Lett. 2008, 101, 116602.

(35) Rinehart, J. D.; Long, J. R. Exploiting Single-Ion Anisotropy in the Design of f-Element Single-Molecule Magnets. Chem. Sci. 2011, 2, 2078-2085.

(36) Branzoli, F.; Carretta, P.; Filibian, M.; Zoppellaro, G.; Graf, M. J.; Galan-Mascaros, J. R.; Fuhr, O.; Brink, S.; Ruben, M. Spin Dynamics in the Negatively Charged Terbium (III) Bis-Phthalocyaninato Complex. J. Am. Chem. Soc. 2009, 131, 4387-4396.

(37) Morita, T.; Damjanovic, M.; Katoh, K.; Kitagawa, Y.; Yasuda, N.; Lan, Y.; Wernsdorfer, W.; Breedlove, B. K.; Enders, M.; Yamashita, M. Comparison of the Magnetic Anisotropy and Spin Relaxation Phenomenon of Dinuclear Terbium(III) Phthalocyaninato Single-Molecule Magnets Using the Geometric Spin Arrangement. J. Am. Chem. Soc. 2018, 140, 2995-3007.

(38) Yamabayashi, T.; Katoh, K.; Breedlove, B. K.; Yamashita, M. Molecular Orientation of a Terbium(III)-Phthalocyaninato DoubleDecker Complex for Effective Suppression of Quantum Tunneling of the Magnetization. Molecules 2017, 22, 999.

(39) Katoh, K.; Yamashita, S.; Yasuda, N.; Kitagawa, Y.; Breedlove, B. K.; Nakazawa, Y.; Yamashita, M. Control of the Spin Dynamics of Single-Molecule Magnets by Using a Quasi One-Dimensional Arrangement. Angew. Chem., Int. Ed. 2018, 57, 9262-9267.

(40) Hofmann, A.; Salman, Z. Tuning the Spin Dynamics of Single Molecule Magnets via Dipolar Interactions. J. Phys.: Conf. Ser. 2014, $551,012055$.

(41) Zhang, L.; Cheng, Z.; Huan, Q.; He, X.; Lin, X.; Gao, L.; Deng, Z.; Jiang, N.; Liu, Q.; Du, S.; Guo, H.; Gao, H.-j. Site- and Configuration-Selective Anchoring of Iron-Phthalocyanine on the Step Edges of $\mathrm{Au}(111)$ Surface. J. Phys. Chem. C 2011, 115, 1079110796.

(42) Komeda, T.; Kim, Y.; Kawai, M.; Persson, B. N. J.; Ueba, H. Lateral Hopping of Molecules Induced by Excitation of Internal Vibration Mode. Science 2002, 295, 2055-2058.

(43) Marinica, M. C.; Raşeev, G. Adsorbates in Phonon Bath: Co on $\mathrm{Cu}(211)$ Surface. Surf. Sci. 2002, 497, 227-238.

(44) Braun, J.; Graham, A. P.; Hofmann, F.; Silvestri, W.; Toennies, J. P.; Witte, G. A. He-Atom Scattering Study of the Frustrated Translational Mode of Co Chemisorbed on Defects on Copper Surfaces. J. Chem. Phys. 1996, 105, 3258-3263.

(45) Loth, S.; Von Bergmann, K.; Ternes, M.; Otte, A. F.; Lutz, C. P.; Heinrich, A. J. Controlling the State of Quantum Spins with Electric Currents. Nat. Phys. 2010, 6, 340-344. 